\title{
INDEPENDENT INNER FUNCTIONS IN THE CLASSICAL DOMAINS
}

\author{
by TOMASZ M. WOLNIEWICZ
}

(Received 2 March, 1986)

1. Introduction. Let $B_{n}$ denote the unit ball and $U^{n}$ the unit polydisc in $\mathbf{C}^{n}$. In this paper we consider questions concerned with inner functions and embeddings of Hardy spaces over bounded symmetric domains. The main result (Theorem 2) states that for a classical symmetric domain $D$ of type I and $\operatorname{rank}(D)=s$, there exists an isometric embedding of $H^{1}\left(U^{s}\right)$ onto a complemented subspace of $H^{1}(D)$. This should be compared with results of Wojtaszczyk [13] and Bourgain [3, 4] which state that $H^{1}\left(B_{n}\right)$ is isomorphic to $H^{1}(U)$ while for $n>m, H^{1}\left(U^{n}\right)$ cannot be isomorphically embedded onto a complemented subspace of $H^{1}\left(U^{m}\right)$. Since balls are the only bounded symmetric domains of rank 1 and they are of type I, Theorem 2 shows that if $\operatorname{rank}\left(D_{1}\right)=1, \operatorname{rank}\left(D_{2}\right)>1$ then $H^{1}\left(D_{1}\right)$ is not isomorphic to $H^{1}\left(D_{2}\right)$. It is natural to expect this to hold always when $\operatorname{rank}\left(D_{1}\right) \neq \operatorname{rank}\left(D_{2}\right)$ but unfortunately we were not able to prove this.

The existence of an isometric embedding of $H^{p}\left(U^{s}\right)$ into $H^{p}(D)$ is closely related to the existence of $s$ independent inner functions on $D$ (Theorem 1). The problem of existence of inner functions on arbitrary bounded symmetric domains has been solved by Aleksandrov's and Løw's constructions for the unit ball [1, 8]. Inner functions on other symmetric domains can be then obtained by a simple averaging argument (cf. [2]). In this paper we construct families of independent inner functions on various classical domains and obtain isometric embeddings of $H^{p}\left(U^{s}\right)$ into $H^{p}(D)$. These constructions depend on [1] but are different than the general construction of one function as in [2].

2. Notation and definitions. Let $D$ be a classical symmetric domain in its standard realization (cf. e.g. [7] or [12]); then $D$ is circular and convex. The Bergman-Shilov boundary $b D$ of $D$ is circular, it is a compact real-analytic submanifold of $\mathbf{C}^{n}$ and it has a unique $\Gamma_{0}$-invariant measure $\sigma_{D}$. $\left(\Gamma_{0}\right.$ stands for the isotropy subgroup of the group of all holomorphic automorphisms of $D$.)

For $0<p<\infty$ the Hardy space $H^{p}(D)$ is defined as consisting of all functions $f$ holomorphic in $D$ and such that

$$
\sup _{0<r<1} \int_{b D}|f(r z)|^{p} d \sigma_{D}(z)<\infty .
$$

For $p=\infty$ the space $H^{\infty}(D)$ consists of all bounded holomorphic functions in $D$. Every function $f \in H^{p}(D)$ has radial limits (denoted by $f^{*}$ ) $\sigma_{D}$-a.e. on $b D$ and when we identify $f$ with $f^{*}$, the space $H^{p}(D)$ can be viewed as the closure of holomorphic polynomials in $L^{p}\left(b D, \sigma_{D}\right)$. The most appropriate reference to this subject seems to be [12].

Classical symmetric domains are divided into four groups. We will only need explicit forms of types I and II. Other types will be only mentioned, the reader is referred to [7]

Glasgow Math. J. 29 (1987) 229-236. 
or [12]. Let $M_{m, n}$ denote the set of all $m \times n$ complex matrices, $M_{n}=M_{n, n} . I$ will stand for the identity matrix of an appropriate size, $Z^{t}, Z^{*}$ for transposed and hermitian conjugate matrix of $Z$. $Z>0$ means that $Z$ is positive definite.

$$
\begin{gathered}
R_{\mathrm{I}}(m, n)=D=\left\{Z \in M_{m, n}: I-Z^{*} Z>0\right\} \quad(m \geq n), \\
b D=\left\{Z \in M_{m, n}: I=Z^{*} Z\right\}, \\
\operatorname{rank}(D)=n, \\
R_{\mathrm{II}}(n)=D=\left\{Z \in M_{n}: I-Z^{*} Z>0, Z=Z^{\prime}\right\}, \\
b D=\left\{Z \in M_{n}: I=Z^{*} Z, Z=Z^{\prime}\right\}, \\
\operatorname{rank}(D)=n .
\end{gathered}
$$

(For the definition of rank see e.g. [6, p. 354].) Note that $R_{\mathrm{I}}(m, n)$ is the unit ball in $M_{m, n}$ with the operator norm and similarly $R_{\mathrm{II}}(n)$ but restricted to symmetric matrices. $b R_{\mathrm{I}}(m, n)$ consists of all unitary embeddings of $\mathbf{C}^{n}$ into $\mathbf{C}^{m}$ and $b R_{\mathrm{II}}(n)$ of all symmetric unitary matrices on $\mathbf{C}^{n}$.

A function $g$ is called inner in $D$ if $g \in H^{\infty}(D)$ and $\left|g^{*}\right|=1$ a.e. on $b D$. By independent inner functions we mean functions $g_{i}$ such that $g_{i}^{*}$ are independent as random variables on $\left(b D, \sigma_{D}\right)$ or equivalently that whenever $\alpha_{1}, \ldots, \alpha_{s} \in \mathbf{Z}$, not all zero, we have

$$
\int_{b D}\left(g_{1}^{*}\right)^{\alpha_{1}} \cdots\left(g_{s}^{*}\right)^{\alpha_{s}} d \sigma_{D}=0
$$

(if $\alpha<0$ then $z^{\alpha}$ means $\bar{z}^{|\alpha|}$ ).

We will use the standard notation: $U$ for the unit disc, $\mathbf{T}$ for the unit circle, $B_{n}, S_{n}$ for the open unit ball and the sphere in $\mathbf{C}^{n} . d m, d m_{n}, d \sigma_{n}$ will be the normalized Lebesgue measures on $\mathbf{T}, \mathbf{T}^{n}, S_{n}$ respectively.

The author would like to thank P. Wojtaszczyk, R. M. Timoney and J. Grabowski for helpful conversations.

3. Inner functions and embeddings. Let $D$ be a bounded symmetric domain and $0<p \leq \infty$. Consider the two conditions.

(a) There exists an isometric embedding $T: H^{P}\left(U^{s}\right) \rightarrow H^{p}(D)$ preserving the constant functions.

(b) These exist $s$ independent inner functions on $D$. We have

THEOREM 1. For every $0<p \leq \infty$ (b) implies (a); if in addition $p \neq 2$ or $\infty$ then (a) implies (b).

Proof. (a) $\Rightarrow$ (b) follows, with only a little work, from Theorem II of [10].

(b) $\Rightarrow$ (a). Put $\Phi: D \rightarrow U^{s}$ equal to $\left(g_{1}, \ldots, g_{s}\right)$. Then (1) guarantees that any polynomial $F$ in $z_{i}$ and $\bar{z}_{i}$ satisfies

$$
\int_{b D} F \circ \Phi^{*} d \sigma_{D}=\int_{\mathbf{T}^{s}} F d m_{s}
$$


By density we obtain the same for all $F \in L^{1}\left(\mathbf{T}^{s}, d m_{s}\right)$. This easily implies that $T f=f \circ \Phi$ is an isometric embedding of $H^{p}\left(U^{s}\right)$ into $H^{p}(D)$.

\section{Domains of type $I$.}

THEOREM 2. Let $D$ be a product of classical symmetric domains of type I and $\operatorname{rank}(D)=s$. Let $1 \leq p \leq \infty$. Then $H^{p}\left(U^{s}\right)$ can be isometrically embedded onto a 1-complemented subspace of $H^{p}(D)$.

As an immediate consequence of main results of [13] and [3] and Theorem 2 we get

Corollary. Let $D \subset \mathbf{C}^{n}$ be as in Theorem 2 and $D^{\prime} \subset \mathbf{C}^{m}$ a bounded symmetric domain of rank one (i.e. $\left.D^{\prime}=B_{m}\right)$; then $H^{1}(D)$ is isomorphic to $H^{1}\left(D^{\prime}\right)$ iff $\operatorname{rank}(D)=1$.

Remark 1. If $p=\infty$, a more general version of Theorem 2, due to R. M. Timoney [11], is known. Namely if $D$ is any bounded symmetric domain with $\operatorname{rank}(D)=s$ then $H^{\infty}\left(U^{s}\right)$ can be isometrically embedded onto a 1-complemented subspace of $H^{\infty}(D)$. It should be however noted that in this case the proof is fairly easy and does not use the inner functions.

Proof of Theorem 2. As there is no difficulty in generalizing Theorem 2 to products of domains we will assume that $D=R_{1}(m, n)$ and treat it as contained in the natural way in $M_{m, n}=\mathbf{C}^{m} \times \ldots \times \mathbf{C}^{m}$.

Let $e_{k}$ denote the $k$ th unit vector in $\mathbf{C}^{n}$. Define $\pi_{k}: M_{m, n} \rightarrow \mathbf{C}^{m}$ as $\pi_{k}(Z)=Z e_{k}$, i.e. $\pi_{k}(Z)$ is the $k$ th column of the matrix $Z$.

LeMmA 1. $\pi_{k}(D)=B_{m}$ and $\pi_{k}(b D)=S_{m}$.

Both equalities can be easily shown using the operator descriptions of $D$ and $b D$.

Now we will show that a counterimage of a set of measure zero by $\pi_{k}$ is still of measure zero.

On any smooth manifold we can define a collection of sets of measure zero without specifying an exact measure, by taking the counterimages of sets of measure zero by the local coordinates. It is easily seen that on the Bergman-Shilov boundary of a symmetric domain sets of measure zero in the above sense coincide with sets of $\sigma_{D}$ measure zero.

Lemma 2. Let $M, N$ be connected real-analytic manifolds and $\pi: M \rightarrow N$ a realanalytic surjection. Suppose $E \subset N$ is of measure zero; then so is $\pi^{-1}(E)$.

Sketch of the proof. We use Sard's theorem, together with the fact that zero sets of real-analytic functions are of measure zero, to show that the set where the derivative of $\pi$ is not surjective is of measure zero. This allows us to omit this set and the rest of the argument is quite simple.

Remark 2. Lemma 2 will be also used in Section 4. In the case of domains of type I there is a more elementary way of proving Lemma 2 and it can be in fact shown that in this case $\pi_{k}$ are measure preserving (i.e. $\sigma_{D}\left(\pi_{k}^{-1}(E)\right)=\sigma_{m}(E)$ ). This can be done, for instance, by showing that $E \rightarrow \sigma_{D}\left(\pi_{k}^{-1}(E)\right)$ is a unitary-invariant measure on $S_{m}$. 
Let $G$ be an inner function in $B_{m}, G(0)=0$. Define

$$
g_{k}=G \circ \pi_{k}
$$

then it immediately follows from Lemmas 1 and 2 that $g_{k \mid D}$ are inner in $D$. We will now prove that they are independent and even satisfy a stronger condition than (1).

Lemma 3. Let $h \in H^{1}(D)$. Then for all $\alpha_{1}, \ldots, \alpha_{n} \in \mathbf{Z}$, such that for some $k, \alpha_{k}>0$ we have

$$
\int_{b D} h^{*} \cdot\left(g_{1}^{*}\right)^{\alpha_{1}} \ldots\left(g_{n}^{*}\right)^{\alpha_{n}} d \sigma_{D}=0 .
$$

Proof. We may assume that $h$ is an analytic polynomial. Let $\alpha_{k}>0$ and for $Z \in M_{m, n}$ define $\tau_{Z}: \mathbf{C} \rightarrow M_{m, n}$ as the multiplication of the $k$ th column by the variable $\lambda \in \mathbf{C}$. It is easily seen that for $\lambda \in \mathbf{T}$ the mapping $Z \rightarrow \tau_{Z}(\lambda)$ is in $\Gamma_{0}(D)$. Hence

$$
\begin{array}{rl}
\int_{b D} h \cdot\left(g_{1}^{*}\right)^{\alpha_{1}} \ldots\left(g_{n}^{*}\right)^{\alpha_{n}} & d \sigma_{D} \\
& =\int_{b D} \int_{\mathbf{T}} h\left(\tau_{Z}(\lambda)\right)\left(g_{1}^{*}\left(\tau_{Z}(\lambda)\right)\right)^{\alpha_{1}} \ldots\left(g_{n}^{*}\left(\tau_{Z}(\lambda)\right)\right)^{\alpha_{n}} d m(\lambda) d \sigma_{D}(Z) .
\end{array}
$$

Notice that for $j \neq k, g_{j}^{*}\left(\tau_{Z}(\lambda)\right)=g_{j}^{*}(Z)$ and $g_{k}^{*}\left(\tau_{Z}(\lambda)\right)=G^{*}\left(\pi_{k}\left(\tau_{Z}(\lambda)\right)\right)=G^{*}\left(\lambda \pi_{k}(Z)\right)$. Hence the right-hand side of (4) can be written as

$$
\int_{b D} \int_{\mathbf{T}} h\left(\tau_{Z}(\lambda)\right) G^{*}\left(\lambda \pi_{k}(Z)\right) d m(\lambda) \prod_{j \neq k} g_{j}^{*}(Z) d \sigma_{D}(Z)
$$

The function $U \ni \lambda \rightarrow h\left(\tau_{Z}(\lambda)\right) G\left(\lambda \pi_{k}(Z)\right)$ is an $H^{\infty}$-function with boundary values $h\left(\tau_{Z}(\lambda)\right) G^{*}\left(\lambda \pi_{k}(Z)\right)$ and vanishing at zero, so the inner integral in (5) must vanish.

Since, obviously (3) is stronger than (1), the operator $T f=f \circ \Phi, \Phi=\left(g_{1}, \ldots, g_{n}\right)$ is an isometric embedding of $H^{p}\left(U^{n}\right)$ into $H^{p}(D)$. Now we will construct a projection onto its image.

Let, for $f \in H^{P}(D)$,

$$
P f=E\left(f^{*} \mid \Phi\right)
$$

be the conditional expectation with respect to the $\sigma$-algebra generated by $g_{1}^{*}, \ldots, g_{n}^{*}$. This operator is a projection with norm equal to one in all $L^{p}\left(b D, \sigma_{D}\right), 1 \leq p \leq \infty$. We will show that $P\left(H^{p}(D)\right)=T\left(H^{p}\left(U^{n}\right)\right)$. Of course, if $f=g \circ \Phi^{*}$ then $P f=f$. On the other hand if $f=P h$ with $h \in H^{p}(D)$ then there exists an $L^{p}$-function $g$ on $\mathbf{T}^{n}$ such that $f=g \circ \Phi^{*}$. We only have to show that $g$ corresponds to an analytic function, or equivalently that

$$
\int_{\mathbf{T}^{n}} g(z) \cdot z_{1}^{\alpha_{1}} \ldots z_{n}^{\alpha_{n}} d m_{n}(z)=0
$$

whenever $\alpha_{j} \in \mathbf{Z}$ and for some $k, \alpha_{k}>0$. But, by (2),

$$
\int_{\mathbf{T}^{n}} g(z) \cdot z_{1}^{\alpha_{1}} \ldots z_{n}^{\alpha_{n}} d m_{n}=\int_{b D}\left(g \circ \Phi^{*}\right) \cdot\left(g_{1}^{*}\right)^{\alpha_{1}} \ldots\left(g_{n}^{*}\right)^{\alpha_{n}} d \sigma_{D}
$$




$$
\begin{aligned}
& =\int_{b D} E(h \mid \Phi) \cdot\left(g_{1}^{*}\right)^{\alpha_{1}} \cdots\left(g_{n}^{*}\right)^{\alpha_{n}} d \sigma_{D}=\int_{b D} E\left(h \cdot\left(g_{1}^{*}\right)^{\alpha_{1}} \cdots\left(g_{n}^{*}\right)^{\alpha_{n}} \mid \Phi\right) d \sigma_{D} \\
& =\int_{b D} h \cdot\left(g_{1}^{*}\right)^{\alpha_{1}} \ldots\left(g_{n}^{*}\right)^{\alpha_{n}} d \sigma_{D}=0 .
\end{aligned}
$$

\section{Other classical domains.}

TheOREM 3. Let $D$ be a product of classical domains of types $R_{\mathrm{I}}(m, n), R_{\mathrm{II}}(n)$, $R_{\mathrm{III}}(2 n), R_{\mathrm{IV}}(n)$ and suppose $\operatorname{rank}(D)=s$. Then there exist $s$ independent inner functions on $D$.

Generalization to products is trivial so we consider only irreducible cases. That of $R_{1}$ is contained in Lemma 3. We will restrict ourselves to the case of $R_{\mathrm{II}}$; the others can be done similarly.

As before we have $D \subset M_{n}=\mathbf{C}^{n} \times \ldots \times \mathbf{C}^{n}$. We will denote variables in this product by $z_{i j}$. It should be noted that $D$ is not open in $M_{n}$ as its dimension is only $n(n+1) / 2$, but it will be of no importance for the proof. Again we define $\pi_{k}(Z)=Z e_{k}$.

Lemma 4. $\pi_{k}(D) \subset B_{n}, \pi_{k}(b D)=S_{n}$.

Proof. Inclusions $\pi_{k}(D) \subset B_{n}, \pi_{k}(b D) \subset S_{n}$ are quite obvious. To show the equality let $z \in S_{n}$. We may assume that $k=1$. If $z=\lambda e_{1}, \lambda \in \mathbf{T}$ then we can take $Z=\lambda I \in b D$ and get $\pi_{1}(Z)=z$. Now suppose $z=\alpha e_{1}+\beta w$ with $\beta>0,\|w\|=1$ and $w \perp e_{1}$. Take

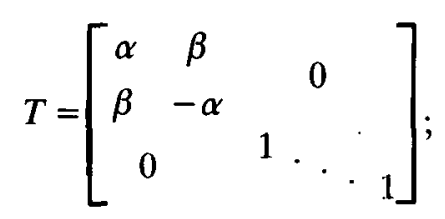

then $T \in b D$. Take a unitary matrix $V$ such that $V e_{1}=e_{1}$ and $V w=e_{2}$; then $Z=$ $V^{*} T \bar{V} \in b D$ and $\pi_{1}(Z)=z$.

In [1] Aleksandrov constructed, for $1 \leq k \leq n$, the inner functions $G_{k}$ such that

$$
G_{k \mid B_{n} \cap\left\{z_{k}=0\right\}} \equiv 0 \text {. }
$$

Take these $G_{k}$ and define

$$
g_{k}=G_{k} \circ \pi_{k}
$$

These functions are well defined on $B_{n} \times \ldots \times B_{n} \subset M_{n}$ and, by Lemmas 2 and $4, g_{k \mid D}$ are inner in $D$. To prove that $g_{k}$ satisfy (1) we will again need an auxiliary mapping $\tau$.

Fix $\alpha_{1}, \ldots, \alpha_{n} \in \mathbf{Z}$ and for $Z=\left(z_{i j}\right) \in M_{n}$ define $\tau_{Z}: \mathbf{C} \rightarrow M_{n}$ as

$$
\left(\tau_{Z}(\lambda)\right)_{i j}= \begin{cases}\lambda z_{i j} & \text { if } \alpha_{i}, \alpha_{j} \geq 0 \\ \bar{\lambda} z_{i j} & \text { if } \alpha_{i}, \alpha_{j}<0 \\ z_{i j} & \text { in other cases }\end{cases}
$$


Also let $\tau_{Z}^{k}=\pi_{k} \circ \tau_{Z}$ and $G=\left\{Z \in b D: \forall_{k} z_{k k} \neq 0\right\}$. $G$ is of full measure in $b D$, for instance because it is a complement of the zero set of an analytic function.

LEMMA 5.

(a) If $Z \in b D$ then $\tau_{Z}(\mathrm{~T}) \subset b D$ and $Z \in G$ implies $\tau_{Z}(U) \subset B_{n} \times \ldots \times B_{n}$.

(b) For every $\lambda \in \mathbf{T}$ the mapping $Z \rightarrow \tau_{Z}(\lambda)$ belongs to $\Gamma_{0}(D)$.

(c) The mapping $Z, \lambda \rightarrow \tau_{Z}(\lambda)$ is continuous on $b D \times \mathbf{T}$.

(d) $\tau_{Z}^{k}$ is holomorphic if $\alpha_{k} \geqslant 0$ and antiholomorphic in the opposite case.

(e) If $Z \in G$ then $\tau_{Z}^{k}$ acts from $U$ into $B_{n}$ and if $\lambda \in \mathbf{T}, r \rightarrow 1$ then $\tau_{Z}^{k}(r \lambda)$ converges to $\tau_{Z}^{k}(\lambda) \in S_{n}$ nontangentially.

The elementary proof of this lemma is left to the reader.

Let $\Phi=\left(g_{1}, \ldots, g_{n}\right), \Phi^{*}=\left(g_{1}^{*}, \ldots, g_{n}^{*}\right)$ with $g_{k}$ being defined by (6). Then $\Phi$ is defined and holomorphic in $B_{n} \times \ldots \times B_{n} \subset M_{n}$. By Lemma 5(a), for $Z \in G$ the composition $\Phi \circ \tau_{z}$ gives a mapping from $U$ into $C^{n}$ and, by (e), we get

If $\lambda \in \mathbf{T}$ is such that for every $k$ the functions $G_{k}$ have the nontangential limits at

$\tau_{Z}^{k}(\lambda)$ then $\Phi \circ \tau_{k}$ has, at $\lambda$, the radial limit equal to $\Phi^{*}\left(\tau_{z}(\lambda)\right)$.

LeMma 6. Let $h \in L^{1}\left(b D, \sigma_{D}\right)$ and $H \subset b D$ be a set of full measure. Then

$$
\int_{b D} h d \sigma_{D}=\int_{H} \int_{\mathbf{T}} h \circ \tau_{Z}(\lambda) d m(\lambda) d \sigma_{D}(Z) .
$$

Proof. Since, for a fixed $\lambda \in \mathbf{T}$ the mapping $Z \rightarrow \tau_{Z}(\lambda)$ belongs to $\Gamma_{0}(D)$, we have

$$
\int_{b D} h d \sigma_{D}=\int_{b D} h \circ \tau_{Z}(\lambda) d \sigma_{D}(Z) .
$$

$\tau$ is continuous in both variables, so after integration of both sides in $\lambda$ we may change the order of integration on the right-hand side and use the fact that $\sigma_{D}(H)=1$.

Now we prove (1). Let $F\left(z_{1}, \ldots, z_{n}\right)=z_{1}^{\alpha_{1}} \ldots z_{n}^{\alpha_{n}}$ and let $E$ be the set of all those $Z \in G$ such that for almost every $\lambda \in T$ the mapping $\Phi \circ \tau_{Z}$ has, at $\lambda$, the radial limit equal to $\Phi^{*}\left(\tau_{Z}(\lambda)\right)$. For $Z \in E$ consider the function

$$
\psi_{Z}=F \circ \Phi \circ \tau_{Z}
$$

By Lemma 5(d) this function is holomorphic and $\left|\psi_{Z}\right| \leq 1$ over $U$, so $\psi_{Z} \in H^{\infty}(U)$. $Z \in E$; hence for almost every $\lambda$ the radial limit of $\psi_{Z}$ coincides with $F\left(\Phi^{*}\left(\tau_{Z}(\lambda)\right)\right.$. Besides $\psi_{Z}(0)=0$ since for every $k$ we have $\tau_{Z}(0)_{k k}=0$ which implies that $g_{k}\left(\tau_{Z}(0)\right)=0$. Hence

$$
0=\int_{\mathbf{T}} \psi_{Z}^{*}(\lambda) d m(\lambda)=\int_{\mathbf{T}} F \circ \Phi^{*} \circ \tau_{Z}(\lambda) d m(\lambda) .
$$

If we show that $\sigma_{D}(E)=1$ then, by Lemma 6 , we are done. So let

$$
K=\left\{Z \in b D: \forall_{k} G_{k} \text { has the nontangential limit at } \pi_{k}(Z)\right\}
$$


Applying Lemma 6 to $H=b D$ and $h=\chi_{K}$ we get

$$
\begin{aligned}
1=\sigma_{D}(K) & =\int_{b D} \chi_{K} d \sigma_{D}=\int_{b D} \int_{\mathbf{T}} \chi_{K} \circ \tau_{Z}(\lambda) d m(\lambda) d \sigma_{D}(Z) \\
& =\int_{b D} m\left(\left\{\lambda \in \mathbf{T}: \tau_{Z}(\lambda) \in K\right\}\right) d \sigma_{D}(Z) .
\end{aligned}
$$

Hence, by (7), for almost all $Z \in b D$ the set of those $\lambda \in \mathbf{T}$ such that $\Phi \circ \tau_{Z}$ has the claimed radial limit is of full measure in $\mathbf{T}$. Now $E$ is just the intersection of the set of all such $Z$ with the set $G$.

\section{Remarks.}

Remark 3. If $D$ is a product of domains of type I, then the fact that $\pi_{k}$ preserve measure (cf. Remark 2) can be used to extend the nonfactorization results of Gowda [5] and Rosay [9] to the case of such $D$. This is done by observing that $g \rightarrow g \circ \pi_{k}$ gives an isometric embedding of $H^{p}\left(B_{m}\right)$ into $H^{p}\left(R_{\mathrm{I}}(m, n)\right)$ and the restriction of a function to the $k$ th column of $Z \in R_{\mathrm{I}}(m, n)$ gives a projection. Then the function $f \in H^{1}\left(B_{n}\right)$ which cannot be written as $f_{1} . f_{2}$ with $f_{1}, f_{2} \in H^{2}\left(B_{n}\right)$ can be "pulled" into $H^{1}\left(R_{\mathrm{I}}(m, n)\right)$.

Remark 4. The proof of Theorem 3 strongly uses the fact that the Bergman-Shilov boundary of $R_{\mathrm{II}}$ consists of unitary matrices; this is also true for $R_{\mathrm{III}}(2 k)$ but not for $R_{\mathrm{III}}(2 k+1)$ and this is the reason why this case is not included.

ReMARK 5. There is a natural question about the maximality of systems of independent inner functions obtained in Theorem 3. This is an open problem even in the simplest case of the unit disc. The situation in this case seems to be much simpler since the existence of two such functions implies the existence of infinitely many.

\section{REFERENCES}

1. A. B. Aleksandrov, The existence of inner functions in the ball, Math. USSR-Sb. 46 (1983), 143-159.

2. A. B. Aleksandrov, Inner functions in compact spaces, Funktsional. Anal. i Prilozhen. 18 (1984), no. 2, 1-13 (in Russian).

3. J. Bourgain, The non-isomorphism of $H^{1}$-spaces in one and several variables, J. Funct. Anal. 46 (1982), 45-47.

4. J. Bourgain, The non-isomorphism of $H^{1}$-spaces in a different number of variables, Bull. Soc. Math. Belg. Sér. B 35 (1983), 127-136.

5. M. S. Gowda, Nonfactorization theorems in weighted Bergman and Hardy spaces on the unit ball of $\mathbf{C}^{n}(n>1)$, Trans. Amer. Math. Soc. 277 (1983), 203-212.

6. S. Helgason, Differential geometry and symmetric spaces (Academic Press, 1962).

7. L. K. Hua, Harmonic analysis of functions of several complex variables in the classical domains (American Mathematical Society, 1963). 223-229.

8. E. Løw, A construction of inner functions on the unit ball of $\mathbf{C}^{p}$, Invent. Math. 67 (1982), 
9. J. P. Rosay, Sur la non-factorisation des éléments de l'espace de Hardy $H^{1}\left(U^{2}\right)$, Illinois $J$. Math. 19 (1975), 479-482.

10. W. Rudin, $L^{p}$-isometries and equimeasurability, Indiana Univ. Math. J. 25 (1976), 215-228.

11. R. M. Timoney, Bloch functions in several variables. II, J. Reine Agnew. Math. 319 (1980), 1-22.

12. S. Vagi, Harmonic analysis on Cartan and Siegel domains, in J. M. Ash, ed., Studies in harmonic analysis (Mathematical Association of America, 1976).

13. P. Wojtaszczyk, Hardy spaces on the complex ball are isomorphic to Hardy spaces on the disc, $1 \leq p<\infty$, Ann. of Math. (2) 118 (1983), 21-34.

TOMASZ M. WOLNIEWICZ

INSTITUTE OF MATHEMATICS

Nicolaus Copernicus University

TORuń, Poland 